\title{
Nuclear envelope rupture in Drosophila D11 cells inhibits
} \section{mitosis}

\author{
Snezhanna Sergeevna Saydakova \\ Sector of Cell Structural Biology \\ ICG SB RAS \\ Faculty of Natural Sciences \\ NSU \\ Novosibirsk, Russia \\ custodian.of.midnight@gmail.com
}

\author{
Ksenia Nikolaevna Morozova \\ Sector of Cell Structural Biology \\ ICG SB RAS \\ Novosibirsk, Russia \\ morozko@bionet.nsc.ru
}

Elena Vladimirovna Kiseleva

Sector of Cell Structural Biology

ICG SB RAS

Novosibirsk, Russia

elka@bionet.nsc.ru

\author{
Gera Alekseevna Pavlova \\ Laboratory of Cell Division \\ $I M C B S B R A S$ \\ Novosibirsk, Russia \\ gera.pavlova@mcb.nsc.ru
}

\begin{abstract}
It is already known that lesions in the organization of the nuclear envelope (NE) negatively affects the stability of the genome functioning, transport of molecules between the nucleus and the cytoplasm, as well as the process of cell division. In previous studies we demonstrated the atypical interaction of ER membranes with the outer nuclear membrane (ONM) at the prometaphase stage, leading to the formation of 4-layered membranes and a disassembly delay of the NE [1]. Our current study is focused on the ultrastructural organization of Drosophila D11 cells, which shows a significant decrease in the number of dividing cells. Our TEM analysis demonstrated that the nuclei of many cells contain numerous microtubules in the nucleoplasm, slightly compacted chromosomes and an atypical NE with extended folds and outgrowths shaped like 4layered membranes. These structures represent membrane complexes consisting of inner nuclear membranes (INM) layers close attached to each other. The formation of multilayer fragments from adherent outgrowths of the NE was previously observed when the lamina protein Lam or the insect-specific protein KUGELKERN was overexpressed [2]. According to these data we suggest that the low mitotic index of Drosophila D11 cells division is associated with misregulation of the mitosis process, possibly due to imbalance in the expression of genes encoding lamina proteins, which stimulates the synthesis and elongation of the nuclear membrane without disassembling it. Excessive NE membranes form loops and folds protruding to the cytoplasm and sticking together due to mutual fusion of the INMs forming the quadruple structures.
\end{abstract}

Keywords - nuclear envelope, endoplasmic reticulum, MLDmD11 cell line, nuclear lamina, Drosophila, TEM

\section{Introduction}

The NE consists of the outer and inner membranes (ONM and INM, respectively) clamped together with nuclear pore complexes attached to the lamina underlying the INM. These are dynamic structures that transform throughout cell cycle stages. The proper organization of the $\mathrm{NE}$ depends on the expression level genes encoding the protein components of the ER, ONM, INM and lamina [2]. Defects in the NE organization with quadruple membranes formation was observed in Hela and C. elegans cells as the consequence of LIPIN and TMEM170 protein level variability, which are ER and the NE proteins, respectively $[3,4]$. In 2018 we observed similar quadruples formed in about $70 \%$ of all membranes in
S2 cells during prometaphase stage [1]. We suggested that these abnormalities could be due to LIPIN and TMEM170 levels misbalance.

The atypical NE organization was shown when lamina proteins were overexpressed in intestinal stem cells and enterocytes of D. melanogaster [2]. In particular, overexpression of Lam led to extra NE membranes formation with small fragments of quadruple membranes formed by the INM contacts with itself.

The aim of the current study was to perform TEM analysis of the NE organization in D11 cells which derive from the eyeantennal disk of $D$. melanogaster. These cells showed very low mitotic index suggesting the presence of defects in the NE dynamics.

\section{Materials and methods}

Cells ML-DmD11 were maintained at $25^{\circ} \mathrm{C}$ in Shields and Sang M3 Insect medium supplemented with 10\% Fetal Bovine Serum (FBS) and $10 \mu \mathrm{g} / \mathrm{ml}$ insulin [6]. Fixation for the TEM analysis performed according to previously described method [5]. Ultrathin sections were made with the diamond knife (Diatome, Switzerland) on the ultramicrotome (Leica ultracut UCT, Vienna, Austria). The sections were observed under the JEOL1400 electron microscope (Jeol, Japan) in the Interinstitutional center for microscopic analysis of biological objects (ICG SB RAS, Novosibirsk, Russia).

\section{Results and conclusions}

Detailed TEM analysis revealed the absence of quadruple membranes formed by the NE and ER stacking on each other similar to those observed in S2 cells. At the same time, we found long quadruple membranes formed by NE folds and loop-like structures (Fig.1). Nuclei with abnormal NE observed in $40 \%$ of all examined cells. As a result, these NE fragments form long protrusion into the cytoplasm suggesting the extra NE growth. The presence of numerous microtubules in the nucleoplasm of these cells as well as slightly compacted chromosomes may indicate that they are paused at the beginning of mitosis. The cytoplasm is characterized by high ribosome density and the presence of some swellings of ER that also implies that these cells are at the beginning of mitosis. It is possible that the nuclei with an excess of NE could havedivision problems probably due to lamina defects. It was previously demonstrated that similar structures can be the result of the lamina protein 
LAM or the insect KUGELKERN overexpression [2]. Presumably, some lamina proteins are overexpressed in D11 cells causing extra NE membranes growth and as a consequence folds of the NE additional fragments due to fusion of INM on itself. To verify this hypothesis, we are going to measure the expression level of genes encoding lamina proteins in D11 cells.

\section{REFERENCES}

[1] A. Strunov et al, "Ultrastructural analysis of mitotic Drosophila S2 cells identifies distinctive microtubule and intracellular membrane behaviors”, BMC Biology, vol. 16, №1, p. 68, May 2018.

[2] R. Petrovsky, G. Krohne, J. Großhans, "Overexpression of the lamina proteins Lamin and Kugelkern induces specific ultrastructural alterations in the morphology of the nuclear envelope of intestinal stem cells and enterocytes", European journal of cell biology, vol. 97, №2, pp. 102-113, March 2018 .

[3] A. Christodoulou et al, "Transmembrane protein TMEM170A is a newly discovered regulator of ER and nuclear envelope

\section{ACKNOWLEDGMENT}

The work was supported by budjet project 032420190042-C-01.

morphogenesis in human cells”, J Cell Sci., vol. 129, №. 8, pp. 15521565, April 2016

[4] A Golden., J. Liu, O. Cohen-Fix, "Inactivation of the C. elegans lipin homolog leads to ER disorganization and to defects in the breakdown and reassembly of the nuclear envelope", Journal of cell science, vol. 122, №. 12, pp. 1970-1978, June 2009.

[5] A. Strunov et al, "A simple and effective method for ultrastructural analysis of mitosis in Drosophila S2 cells", MethodsX, vol. 3,.pp. 551559, October 2016

[6] https://dgrc.bio.indiana.edu/product/View?product=94

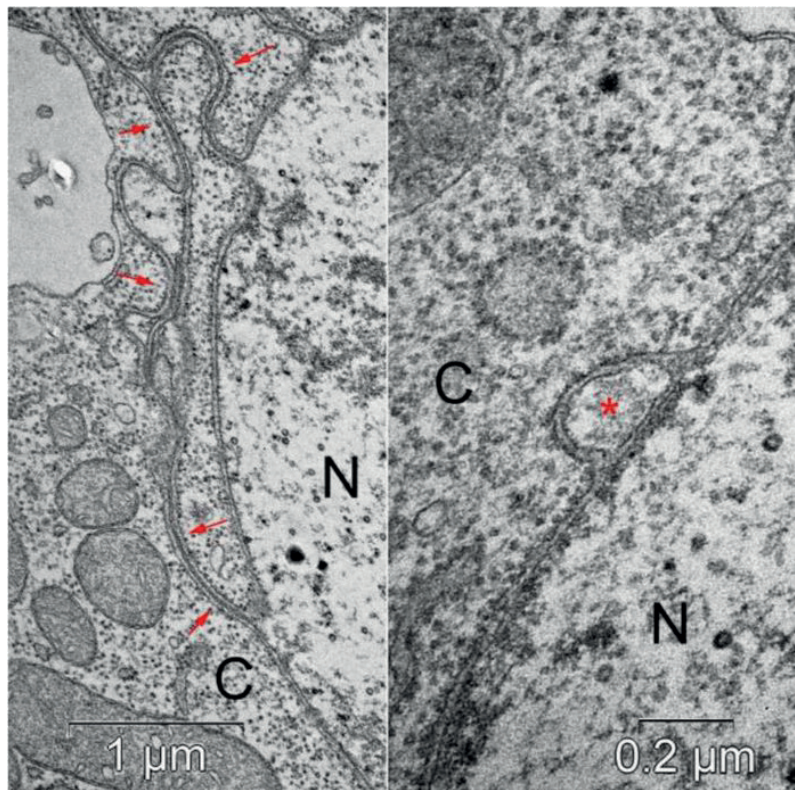

Fig. 1. Abnormal NE in D11 cells. Arrows shows the NE fragments stacked on each other; the loop-like structure is shown with asterisk. $\mathrm{N}$ - nucleus, $\mathrm{C}$ - cytoplasm. specific protein 